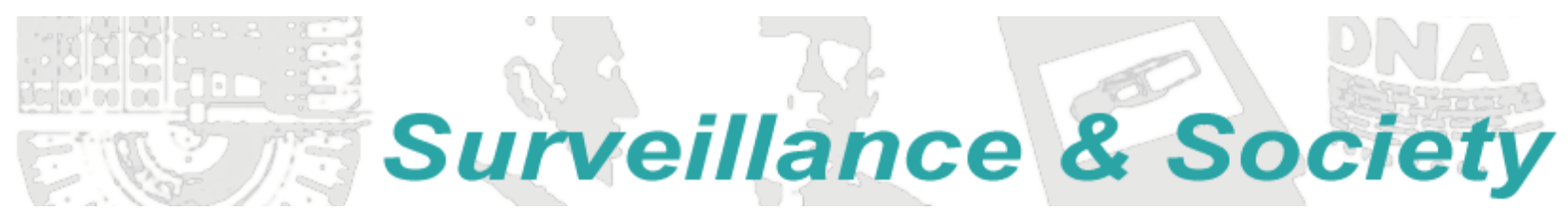

\title{
Article $\quad$ Governing the Wild: Databases, Algorithms, and Population Models as Biopolitics
}

\section{Irus Braverman}

Cornell University, USA.

ib266@,cornell.edu

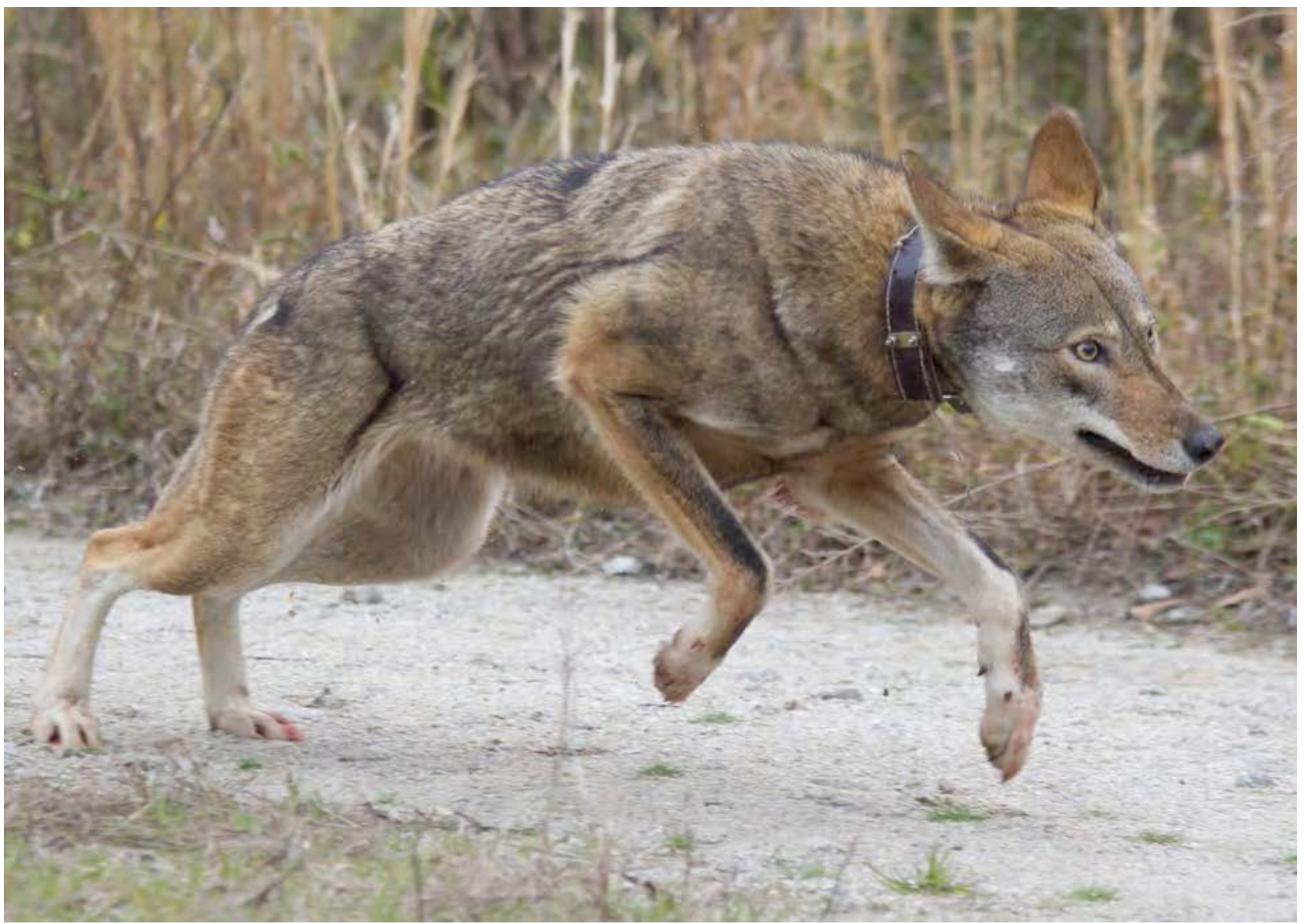

Figure 1: Female red wolf 1470 was one of a litter of five pups born in Point Defiance Zoo \& Aquarium in 2006. Thirteen days later, she and a male and female sibling were placed in the den of a wild female in Tyrrell County, North Carolina, for foster rearing. Since then, 1470 has raised two litters in the red wolf recovery area. Rebecca Bartel/USFWS took this photo in early 2013, after 1470 was fitted with a new radio collar and released. Used with permission.

Braverman, I. 2014. Governing the Wild: Databases, Algorithms, and Population Models as Biopolitics. Surveillance \& Society 12(1): 15-37. http://www.surveillance-and-society.org I ISSN: 1477-7487

(c) The author(s), 2014 | Licensed to the Surveillance Studies Network under a Creative Commons Attribution Non-Commercial No Derivatives license. 


\begin{abstract}
This essay draws on interviews with conservation biologists to reflect on two interrelated aspects of the in situ - ex situ divide and its increasing integration: database systems and population management models. Specifically, I highlight those databases and software programs used by zoos in ex situ conservation settings, and the parallel, traditionally distinct, in situ databases and risk assessment models. I then explore the evolving technologies that integrate wild-captive databases and population models and, in particular, emerging metapopulation and meta-model approaches to small population management. My central argument is that, while still viewed by many as separate, the in situ and ex situ projects - and their respective elaborate administrative structures and models of calculationare, in practice, increasingly bleeding into one another. The stories I tell here about the efforts to save the red wolf from extinction reveal the complexities of this integration. I also document how-in this process - a tiny group of experts translates data into algorithmic formats to generate standardized risk calculations that are meant to apply both universally and objectively. Applying Foucauldian and STS insights to the field of conservation biology, I argue, finally, that surveillance and biopolitics work hand-in-hand in this context to enable a comprehensive, effective, and unitary management of nonhuman population life, or "viability".
\end{abstract}

No longer can the zoo world operate differently from a national park. We have to be working as a partnership because we need each other. The captive populations are most likely not sustainable on their own and don't have a purpose on their own and the wild population certainly is not viable on its own either. So different groups and disciplines need to be working together.

Robert (Bob) Lacy, population biologist, interview

\title{
In Situ - Ex Situ: An Introduction
}

Although understudied in the social sciences and mostly unheard of by the general public, the in situ - ex situ dichotomy has arguably shaped - and still very much shapes - the development of conservation and its institutional alliances, especially in its particular manifestation in conservation biology over the last few decades (Braverman 2015). Some conservationists have suggested, accordingly, that "anyone who has taken a course in conservation biology, or even read a book on the subject, knows that there are fundamentally two kinds of conservation: in situ and ex situ," which are Latin for "in" and "out" of place (Burney and Burney 2010: 16). Scientists, academics, and administrators are currently debating the importance of keeping the in situ - ex situ terminology alive, some claiming that, without it, the very meaning of conservation collapses, others arguing that its use is dangerously misleading and that it should therefore be declared dead (Redford et al. 2013). Lurking beneath the surface of this dispute are the older and seemingly less scientific cousins of the in situ and ex situ distinction: nature versus culture and wilderness versus captivity (Braverman 2014a).

This essay will draw on interviews with conservation biologists as well as participant observations and secondary data, to reflect on two interrelated aspects of the in situ - ex situ divide and its emergent integration: database systems and population management models. Specifically, I will highlight those databases and software programs used by zoos in ex situ conservation settings, and the parallel, formerly distinct, in situ databases and risk assessment models. I will then explore the evolving technologies that integrate wild-captive databases and population management and, in particular, emerging metapopulation and meta-model approaches to wildlife management.

Such new database management techniques and population models may very well offer a way out of the problematic nature-culture schism (for more about this schism, see Braverman 2014a). At the same time, however, integrative management processes also further the pervasiveness of what are framed as global, unitary, scientific, and apolitical logics. Performed by a small group of experts, the science of small 
population management inserts data into algorithmic formats to generate standardized risk calculations that are claimed to apply both universally and objectively. When represented within a closed algorithmic system, the unknown and undetermined is supposedly harnessed into the familiar and predictable. But herein lies the tension: the differentiation and fragmentation of management apparatuses and actual populations is all too often the result of projections of the controversial nature/human divide, which can be detrimental to conservation goals. At the same time, treating such populations as a single unit for global management-as frozen peas to be shuffled around-can problematically erase the historical, geographical, ecological, social, and political contexts in which these populations live, interact, and dieas well as the differences between them - thereby contributing to their alienation and to their demise.

The stories I tell here about the dramatic efforts to save the red wolf from extinction, along with many other stories that I tell elsewhere about other species (Braverman 2015), reveal the complexities of this mission. My central argument is that, while viewed by many as separate, the previously distinct in situ and ex situ projects - and their respective elaborate administrative structures and models of calculation-are increasingly bleeding into one another. I will trace these developments through Foucault's surveillance and biopolitical frameworks and will interpret them in light of Science and Technology Studies' (STS) emergent studies on the prevalence of algorithms. Although the application of both frameworks to nonhuman populations remains highly contested, I argue here that such an application is both relevant and important.

\section{The Application of Surveillance and Biopower to Nonhuman Populations}

Michel Foucault uses the term biopower to describe the project of governing human bodies, populations, and life. Whereas Nikolas Rose, alone and with Paul Rabinow, argues for the inapplicability of biopower in the nonhuman contexts, my project draws on scholars such as Donna Haraway (2008), Cary Wolfe (2013), and Nicole Shukin (2009) to argue that biopower is also an important conceptual framework for nonhumans. This, arguably, is acutely relevant in the context of conservation (Youatt 2008; Holloway and Morris 2012; Lorimer 2012). I have argued elsewhere for the applicability of Foucault's surveillance and care to the context of nonhuman animals (Braverman 2012a, 2012b). Kevin Haggerty and Daniel Trottier have argued more broadly that, "Nature is, by degrees, becoming nature under observation" (Haggerty and Trottier 2013: 6). They accentuate how " "natural' domains are increasingly suffused with human-initiated and coordinated surveillance measures, and how the ostensibly "unnatural" world of humans is becoming permeated by surveillance initiatives with diverse connections to nature" (2013: 2). Elsewhere, I have suggested that we consider conservation without nature - in its pristine form at least (Braverman 2014b; see also Braun 2006; Lorimer 2012). By challenging the bifurcation of nature and human, wild and captive, and in situ and ex situ conservation, I have problematized some of the central assumptions of conservation biology (Braverman 2015). I will not repeat these claims here, but will argue that they can be situated within the biopolitical framework. When harnessed for the project of "making life," surveillance - here, in the form of extensive records and data management —works hand-in-hand with biopower-here in the form of translating the data into risk modalities for populations and programs for operation.

More generally, Donna Haraway has long argued that imposing human boundaries onto practices of biopower is yet another form of human exceptionalism (Haraway 2008: 53). Alongside her rejection of the notion that nonhuman animals cannot be subjects, Haraway asserts that animals, humans, and technologies are intimately entangled in scientific practices in ways that expose the traffic of knowledge across the permeable human-nonhuman species divide. Similarly, Carrie Friese considers how cloning endangered animals "is embroiled in a biopolitics that links humans and animals through both the traffic in techniques and in corresponding human-animal relations through which both species become together" (Friese 2013: 14). She asks in this context: "Can we still use the term 'wildlife' to denote animals that may not be 
tamed, but have nonetheless been remade from the inside out by humans in order to fit the planet as it exists today?" (Friese 2013: 91).

Finally, in Against Ecological Sovereignty, Mick Smith argues that "the implications of biopolitics for ecology and the ecological implications of biopolitics have hardly ever been noticed" (Smith 2011: xv). This essay responds to Smith's concern by expanding Foucault's biopolitics into the relatively new field of conservation biology. I will show how surveillance and biopolitics work in tandem in the conservation context to enable a comprehensive, effective, and unitary management of nonhuman population lifewhich is referred to by conservation biologists as viability.

Coupled with extinction (the measurement of a population's death risk), the concept of viability instructs the biopolitical project of wildlife population management. While it was developed in both in situ and ex situ contexts in the mid-1980s, the term viability has taken on a different meaning in each context. "There are multiple views on viability," the population biologists I interviewed have explained to me. Phillip Miller is senior program officer for the Conservation Breeding Specialist Group (CBSG) of the International Union for the Conservation of Nature (IUCN). Miller tells me in an interview that although he calculates viability every day, it is a convoluted concept. There are various ways to calculate viability, he tells me, asking rhetorically: "Are you talking about numbers of adults? Are you talking about the growth rate of the population being above a certain threshold? Are you talking about the number of adults being observable in the population over a specified period of time?" Jonathan (Jon) Ballou, a population biologist at the Smithsonian Institute, is one of the masterminds behind the science of small population management, which was developed to address the particular perils of endangered animal populations in the wild. He speaks more generally to the relationship between viability and extinction, insisting that the two are not two sides of the same coin. In his words: "It's like asking: are you healthy or are you dead? Viability's got to do with your health; extinction is death" (interview). According to Ballou, then, viability is much broader than a low risk of extinction.

\section{Zoo and Wild Database Systems}

In situ and ex situ experts have developed two generally discrete systems for identifying, recording, and processing data for managing wildlife populations. This section will explore these two database systems and their respective foci.

Ballou explains the difference between in situ and ex situ in the context of data management. First, he characterizes the data on zoo animals. "We know the details of individuals in captivity," he tells me, "because they're individually marked and because we know their birth dates and death dates." "In zoos, all animals are monitored," Ballou explains. As a result, he continues, "We have identified what information is needed for their management. Data standards have been developed and software has been developed [with] specifics that deal with those kinds of data." In captivity, then, experts have been able not only to identify the data required for standardization but to also obtain it. My book Zooland (2012a) details the various databases operative in zoos, from studbooks, through ARKs, MedARKS, and SPARKS and, most recently, the Zoological Information Management System (ZIMS) that incorporates all extant databases into one global and standardized system. A massive record system coordinated by the zoo's registrar documents the medical and husbandry record of every zoo animal, often on a daily basis.

Yet similar information about in situ animals is harder to come by. Ballou explains that in the wild, "you don't know individuals: you don't see when they're born, you don't see when they die. They just disappear-you don't know if they're dead or if they've left. So it's a much more complicated system to document." Such major differences in the type and amount of data that can be collected between captive and wild settings have resulted in the configuration of separate database systems for their management. 
Population biologist Karin Schwartz tells me in an interview about the relationship between the two database administrations. "There never was a link between captive and wild data management," she explains. The differences are most apparent, Schwartz continues, when a particular animal is transferred from one setting to another. In her words: "Once a captive animal is released into the wild, as part of a reintroduction program, for instance, there is usually no way to track it there." "Right now there is no link and there is very poor communication between the people that are monitoring them in the wild and their records from the people who took care of them in captivity," Schwartz explains. Accordingly, when a captive animal is transferred from captivity-where it is intensively tracked, documented, and recorded in studbooks and international database systems (Braverman 2012a) - into the wild, this animal disappears, only to reappear again on the other side of the divide, this time as a wild animal that can only be loosely surveilled within the constraints of in situ data management.

The excel spreadsheet is a central documentation technology of in situ management. According to Schwartz, field conservationists often "have six different excel spreadsheets for different aspects [of the animal]: one is for blood values, one is for capture events, one is for chemical checks." "So you can't look at an animal [in one database] and find out everything that's happened to it," she says. "You have to look at one animal in this spreadsheet and then to find it in that spreadsheet" (interview). By contrast, ex situ managers have replaced the pen-and-paper spreadsheets with digital databases: a central computer administration that takes data management to more sophisticated and abstract levels. This configuration also renders the data more easily translatable to algorithmic formulation.

Only a few decades back, wild animals were typically not identified, documented, and managed in such an intense fashion. Because of insufficient data, it was also nearly impossible to carry out reliable projections of viability rates. As I have explained elsewhere, this loose form of in situ conservation management was not only a result of the technological limitations of the time, but also ideological: whereas the traditional idea of wildlife conservation was of preservation and protection (in certain variations, "fortress" conservation), in recent decades conservation has evolved into a biopolitical project (Braverman 2015). This transition has manifested in the intensification of in situ management, which has in turn necessitated more comprehensive and accurate databases. Accordingly, digital databases that were previously used only in captivity are increasingly implemented toward more effective management of in situ wildlife populations.

The golden lion tamarin is a good example for the bleeding of database management strategies from zoos into the wild. The software program SPARKS (Single Population Analysis and Records Keeping System) was developed by zoos as a record-tracking device for studbook keepers. Ballou tells me that:

In the golden lion tamarins example, a subset of the wild population was very carefully followed. And so we actually applied SPARKS to collect data. . . . We used it to create life tables, to monitor the pedigrees of the animals, and their relationships; we've used it to determine movements of animals between groups; we've used it to monitor the changing size of the population. The information first went into field notes, and then into SPARKS.

\section{(Ballou, interview)}

Despite these changes, in situ and ex situ systems still differ not only in the types of information collected about populations and the database systems into which this information is fed, but also in how the respective experts collect data in the first place. The next section will examine technologies for collecting data, especially in situ, where such data is usually less readily available. 


\section{Collecting Data}

In Zooland (2012a) I discuss at length the ways in which zoo animals are identified, named, recorded, and thereby governed through their naturalization. This section will focus on how the data is recorded and collected on the other side of the divide: in the wild. Alongside excel spreadsheets, in situ experts (often referred to as "field scientists") have been using Global Positioning System (GPS) and Very High Frequency (VHF) collar technologies to document the movement of the otherwise invisible animals across large territories. Such invisibility is typically irrelevant in captive settings, where the animals are confined and thus could be, and sometimes are, surveilled "24/7," without resorting to GPS or any other sophisticated technology except a camera, perhaps (Braverman 2012a).

Whereas the uses of radio and satellite telemetry technologies are revealing relatively more information about animal movement in the wild, they are at the same time limited: collars fall off or stop transmitting signals after six months, and their signals are often delayed so that by the time the surveyors reach the location the animals have already moved on (Waddell, interview). Such radio collar devices are also limited in that they operate on batteries, whose weight restricts the size of the animal that can be tracked (Smyth and Nebel 2013: 3). External tags (e.g., ear and leg bands, dart tags, painted marks, scale clippings) have also facilitated animal tracking. While these are relatively cost-effective, their external location on the animal's body makes them susceptible to damage; also, they can be illegible upon recapture. Such and other issues have posed significant obstacles to data collection in in situ settings.

Increasingly, however, new technologies are being developed and implemented for optimizing knowledge about in situ animal populations with less and less disruption to their behavior. For example, the Passive Integrated Transponder (PIT) tag is a tiny barcode inserted into individual animals that enables managers to track them during their lifetime. Internal PIT tags were initially used by scientists in the 1980s for measuring the movement of fish. Since then, it has expanded to include research on the movement of a variety of taxa, both in the wild and in zoos (Smyth and Nebel 2013). Because PIT tags only turn on when they are activated by a low frequency radio signal, they require very little in the way of battery power (ibid.). When activated, the PIT tag projects an alphanumeric code back to the reader, who can then use it to track the animal (ibid.).

"Capture-Recapture" is yet another strategy developed by wildlife managers to overcome the problem of unknown data for estimates of population size. This method deducts the known from the unknown population based on the ratios of marked to unmarked individuals. The most famous early application of capture-recapture was Pierre Simon Laplace's estimate of the size of France's human population in 1802 (Polar Bear Specialist Group 2009). More recently, the capture-recapture assessment has been providing demographic assessments of polar bears worldwide. "Now, it's not quite that simple," says Steven Amstrup, director of Polar Bear International, who has been promoting this method for polar bear research. "But here," he explains, "is the idea: calculating the proportion of marked animals to unmarked sample, and then to the unmarked animals in a second sample, gives you information about the ratio of marked animals to the total population" (Amstrup, interview). For example, he continues: "if in one year you capture and mark 100 bears and in the following year you capture another 100 bears in the same area and 10 of them are marked-you might conclude that one in ten bears in the population was marked. Because the total sample size was 100 bears, the total population size could be estimated as $\mathrm{N}=1000$." Through this process, the unknown, too, is harnessed toward calculating the unknown.

In North America, much of the data collected by scientists on species in the wild is standardized by database managers and incorporated into one database: NatureServe. NatureServe's dataset on at-risk in situ populations includes more than 900,000 location records from a network of "biological inventories" operating in the United States and most of Canada. From this organization's materials: "For nearly four decades, this network has collected and managed detailed local information on plants and animals of 
conservation concern and has become a leading source for information about North America's endangered species" (NatureServe 2011). "We have over 60,000 species that we try to keep our finger on the pulse of," Leslie Honey, Vice President of Conservation Services at NatureServe, tells me in an interview. "All of our programs have data managers, [who] work together with the scientists to populate the databases," she says. Data about an animal population comes to stand for the actual population when "populated" into databases by scientists through standardization.

Standardization is the magic word. "Standards," Honey tells me, "are the only way that we're able to aggregate the data and use [it] across jurisdictions." "Otherwise," she continues, "you're comparing apples and oranges, and that's not very helpful. It's tasty, but not helpful!" The application of a generalizing process thus translates local data into units that can in turn be read and compared by experts that are removed from the particular sites. When I ask Honey how information about captive or semi-captive populations is incorporated into the NatureServe databases she pauses. "We have so much work to do with tens of thousands of species in the wild," she finally replies, "that we don't do this stuff." "Stuff" is Honey's term for documenting captive species. This, then, is yet another example for how certain databases are still both determined and constrained by the traditional in situ / ex situ schism.

\section{Integrating Databases: The Case of the Red Wolf (Canis rufus)}

In many other instances, however, the long-standing alienation between wild and captive databases is gradually being bridged. It is no coincidence that the most recent version of the new zoo database system - the Zoological Information Management System (ZIMS) version 3-is being developed so that it can record data not only for zoo animals but also for animals in the wild. Schwartz explains that "ZIMS will put everything in one place" (Schwartz, interview). This technological capacity is arguably part of a broader transition to integrate in situ and ex situ information into a unified and global database system. Animal databases constitute "working archives" and, with ZIMS 3, are shifting toward a "planetary management” (Bowker 2000: 644-5).

The red wolf (Canis rufus) recovery program provides an apt example of the gradual merging and unification of in situ and ex situ administrations, especially with regard to databases. Once occurring throughout the eastern and south parts of central United States, the red wolves were decimated by predator control programs as well as by habitat loss. Red wolves were already listed as endangered in 1967 and are currently considered one of the most endangered canids in the world (Fish and Wildlife Service 2013). In the 1970s, the U.S. Fish and Wildlife Service (USFWS) initiated efforts to locate and capture as many red wolves as possible for the purpose of establishing a captive breeding program with the intention of later reintroducing the species into a portion of its former range. More than 400 canids were captured. However, morphological characteristics determined that of these wolves, only 17 were "pure" red wolves, of which 14 were capable of reproducing. This group of 14 red wolves became what biologists call the "founders" of the entire red wolf population in existence today. By 1980, red wolves were considered "biologically extinct," with the sole surviving wolves housed in captive facilities (Waddell, interview, Braverman 2014b).

William Waddell of Point Defiance Zoo \& Aquarium in Washington coordinates the Red Wolf Species Survival Plan (SSP), which encompasses 44 North American zoos and wildlife centers. The SSP collaborates with the USFWS to manage both zoo and free-ranging red wolves. The red wolf recovery program, the name of this joint venture, was initiated in the early 1970s and, as such, was one of the earliest recovery programs to include zoo-based breeding and management as a strategy for species recovery-preceding the black-footed ferret program and even the Californian condor's (Waddell, interview). Waddell describes the relationship between the two populations: "Zoo animals are the safety net: a fine balance must be struck in the zoo population between supporting the wild population and maintaining a genetically diverse and stable captive population" (interview). 
In 1987, the first four pairs of red wolves were reintroduced from captivity into North Carolina. The process was not easy: "Some of them had to be returned to their enclosures because they were too visible or tolerant of people ... . not knowing what they were supposed to do" (Waddell, interview). Such "unruly" wolves were either replaced or reacclimated. "They were recaptured, and then there was some experimenting," Waddell tells me. "What if we put this pair together in an acclimation pen. We'll allow them to breed, and if they have pups, we'll open up the enclosure gates and see if it's going to keep the pair more localized to the area where they were acclimated."

Overall, approximately 80 wolves have been reintroduced from the zoo population. "Some of them died, some of them had to be brought back to the zoo population, and some- - [we] don't know what happened to them," Waddell tells me. Nonetheless, the wolf population has grown over time and the recovery area has expanded to include three national wildlife refuges, a bombing range, and state and private landsencompassing a total of approximately 1.7 million acres (Red Wolf Recovery Program 2013). In 2013, the red wolf population amounted to 199 animals in captive institutions across the United States and an estimated 100 to 120 animals in eastern North Carolina (Waddell, interview).

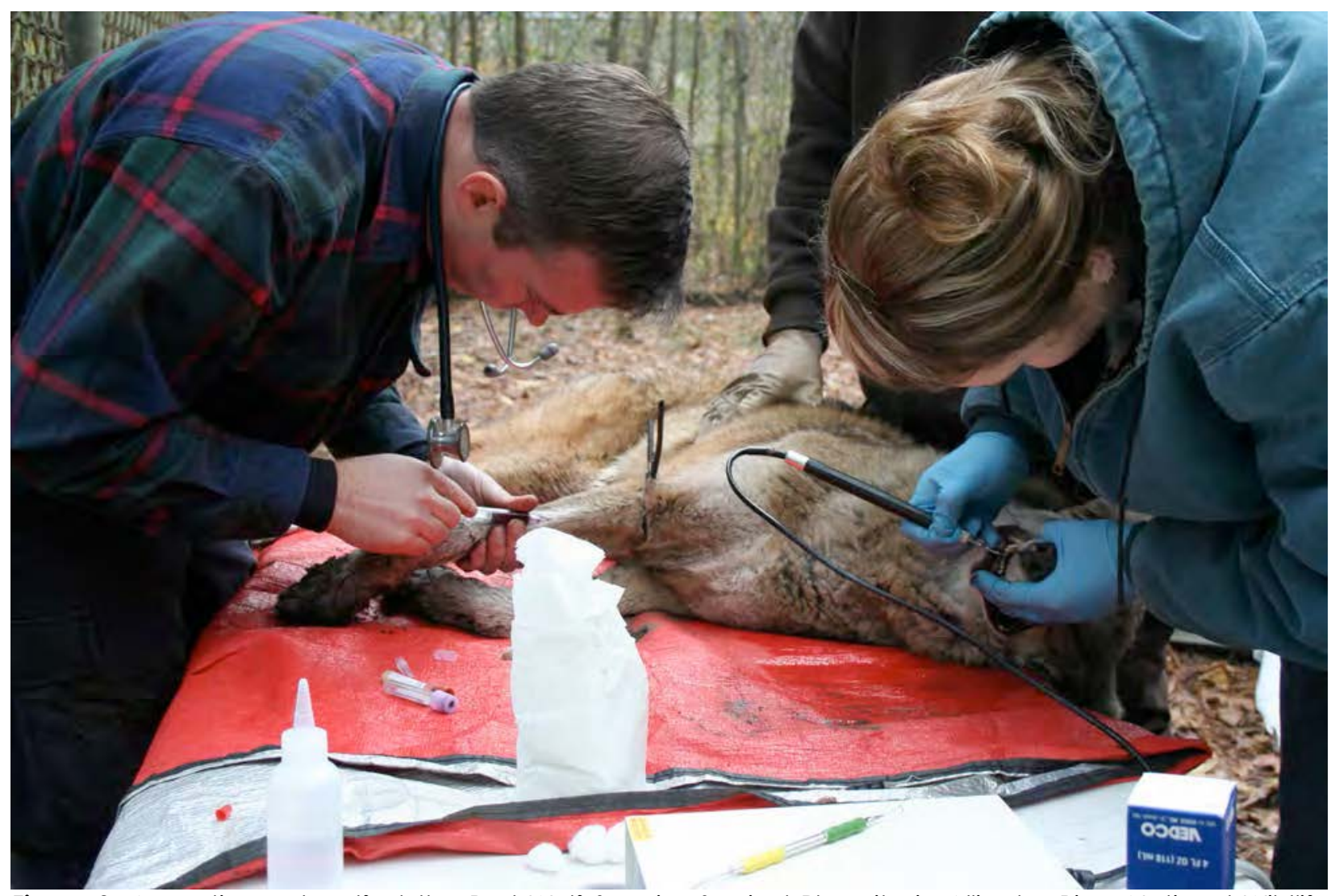

Figure 2: A captive red wolf at the Red Wolf Species Survival Plan site in Alligator River National Wildlife Refuge. The wolves are examined annually, vaccinated, and assessed for medical care. Photo by Ryan Nordsven/USFWS, December 11, 2009.

Although large-scale reintroductions were discontinued in the early 1990 s, constant interactions and exchanges occur between the wild and captive red wolf populations as well as between their respective human managers. "There's no black and white," Waddell tells me. Both in situ and ex situ red wolf populations are closely monitored, he says (see, e.g., Figures 1-4). Captive and wild red wolves alike are given a studbook number and blood samples are taken from all wild-born pups for paternity testing. "We might supplement by fostering one to three pups from the litter in a zoo and put them in the wild den for rearing," Waddell continues. "I wonder what the [mothers] think," he contemplates, "or if they can count. 
But their maternal instincts take over, and it's been successful." For such reintroductions to succeed, field and zoo experts need to be working closely together, Waddell explains. In his words: "If there's any sort of exchange between the zoo population and the wild population, it's based on conversations and discussions between myself and the red wolf recovery biologists at the USFWS."

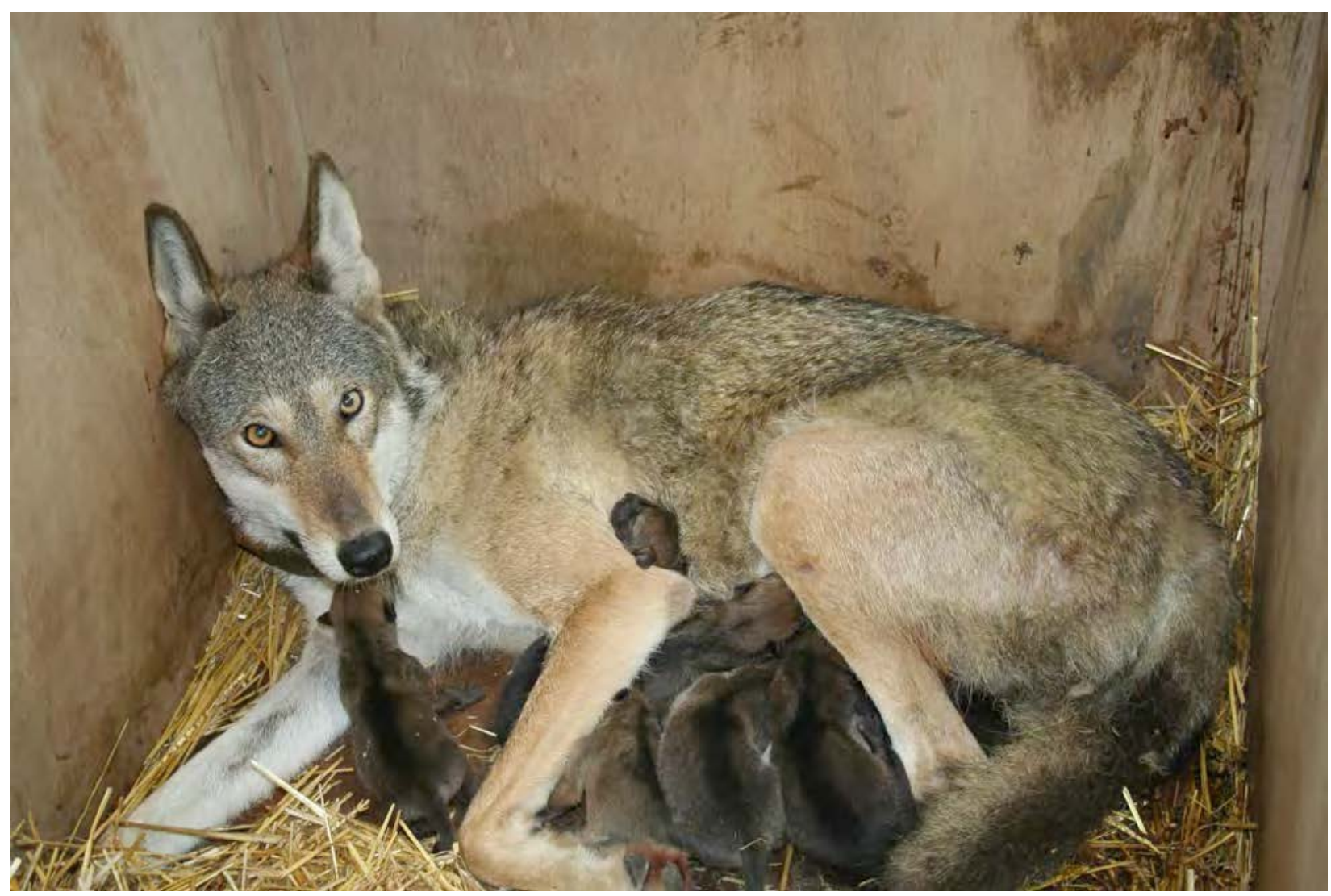

Figure 3: Pups born to $1517 F$ (studbook number, female) in captivity in the Red Wolf Species Survival Plan site, Alligator River National Wildlife Refuge. Photo by Ryan Nordsven/USFWS, April 29, 2008.

A variety of wildlife management efforts thereby create a complex everyday "conviviality" (Hinchliffe and Whatmore 2006) between captive and wild red wolves, eroding the previous administrative and geographical boundaries between them. At the same time, the management of red wolves is still performed by two separate administrative bodies: one in the field, the other in captivity. A 2013 joint initial workshop facilitated by the Lincoln Park Zoo used PVA modeling (see herein) to discuss "database validation" along the in situ / ex situ divide. Currently, efforts are underway to formalize the population management model that has emerged through the collaborative process. 


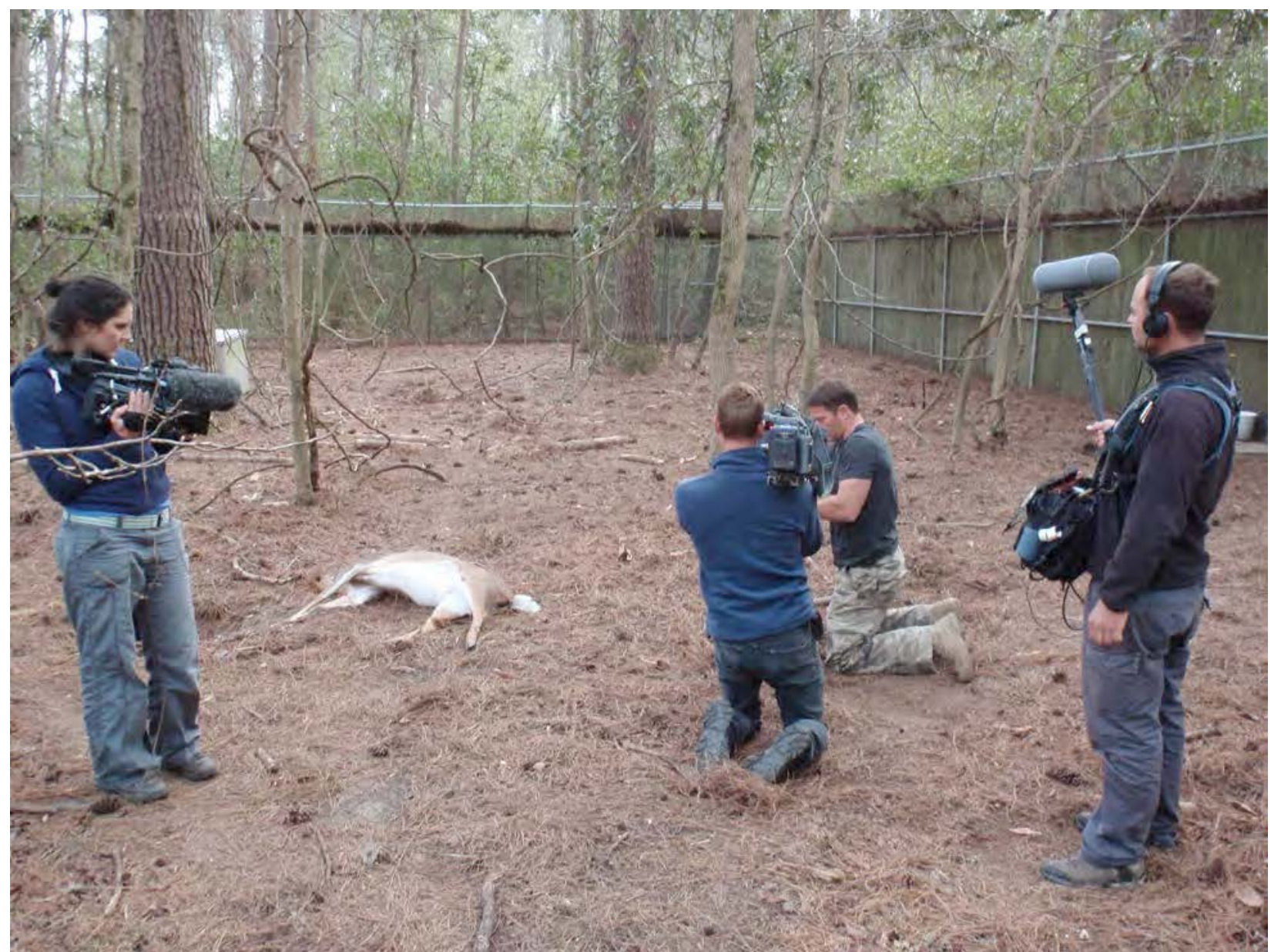

Figure 4: A film crew from the BBC filming the "Deadly Pole to Pole" series visited the Red Wolf Recovery Area in Alligator River National Wildlife Refuge in fall of 2012. In the photo, they were shooting footage of "predation" with a captive pair of red wolves and a roadkill white-tailed deer. Photo credit: M. Morse/USFWS.

\section{Population Management Models: Ex Situ}

Alongside the new database conglomerates and related software models that are mushrooming by the day for integrating databases for in situ and ex situ populations, hybrid computer models are also proliferating for population management. Here, too, the starting point has been the stratification between wild and captive populations, with the practices increasingly shifting toward an integration of the two systems. Good data is not enough, explain population biologists; this data also needs to be aggregated and translated into an operational scheme. To generate such a scheme, zoo experts have designed and used computerized data assessment tools. The major computer programs used by North American zoos are ZooRisk, PM2000 and PMx.

ZooRisk was developed by the Lincoln Park Zoo; it provides a quantitative assessment of a captive population's sustainability based on demographic, genetic, and management processes (Faust and Earnhardt 2008). ZooRisk starts off with a studbook database containing every individual animal's genealogy, age, breeding history, and restrictions. Using this data, the program projects the future of the population at year-by-year intervals based on probabilities of death and reproduction for each individual, up to hundreds of years into the future. ZooRisk users can simulate a variety of breeding management options: they can minimize inbreeding, prohibit genetically detrimental pairs, reach a target population 
size, and allow imports or exports to and from the population. Management decisions are generated by running hundreds of random simulations and observing the range of likely possibilities. Through such numerous repetitions the data on the individual unit is transformed into knowledge about the population.

As solid as these projections may seem to the untrained eye, a closer look at the language of these programs reveals the fragility of their assessments. For example, ZooRisk's manual highlights the importance of data quality: "For populations that are small and/or have historically been small, it is possible that demographic rates cannot be accurately determined, and you should seriously consider the appropriateness of running analyses with poor quality data" (Faust and Earnhardt 2008). For this reason, the American Association of Zoos and Aquariums (AZA) dedicates significant effort to ensure data quality and advises regional studbook keepers on their data preparation for population modeling and management.

However, such models are not only dependent on high data quality; they are also predicated upon the assumption that one can derive all relevant knowledge from standardized data that then translates into algorithmic abstractions. This "trust in numbers" (Porter 1995) is nowhere more evident than in the calculation of extinction risks by software models for population management.

\section{Viability}

The first formal definition of viability was developed by a working group at the National Zoo in Washington DC tasked with codifying the goals of population management in zoos. Ballou tells me that this group came up with a genetic diversity measure for calculating the viability of captive populations. "They imposed this rule that each genetic population should be large enough and robust enough to maintain 90 per cent of the genetic diversity for two hundred years," Ballou tells me. "Not too long after that," he adds, conservation biologists "realized that, "Gosh, it's going to take much too many animals to do that, hundreds and hundreds of animals' - so they changed the goal to 90 per cent for one hundred years." "And that kind of stuck," he adds (Ballou, interview). In the context of zoo management, viability has typically been viewed as synonymous with sustainability (Shaffer 1987; Faust, interview). Because zoo populations have traditionally been perceived as body-doubles of their conspecifics in the wild, the central threat in the context of zoo management was perceived to be inbreeding and impurity, not extinction.

Around the same time that the definition of viability materialized in zoos (mid-1980s), Population Viability Analysis (PVA) emerged as the central mode of analysis for many in situ small population management models. The PVA analysis systematically evaluates the extinction processes that operate on small and often fragmented threatened species by predicting the probable consequences of various management decisions for the viability of the wild population. Robert Lacy explains that "population modeling in the past 25 years has been species-focused: you plug in all the biology of that species and you determine how well that species is doing" (Lacy, interview). Caroline Lees further explains: "when we think about population viability, we think about all the threats to a population - all the things that might reduce it in numbers towards zero, which is extinction." "What we're interested in," she explains, "is how big the population needs to be to keep going" (Lees, interview).

Population biologists attach heightened importance to population size. This, for a variety of reasons - and, mainly that, as a result of what population biologists refer to as stochastic (or random) events, smaller populations are more likely to become extinct than larger ones. The IUCN Red List of Threatened Species - the world's most important classificatory system for at risk nonhuman species-defines the threshold population size as one of three criteria for estimating the conservation status of species. This idea of a threshold population size below which a species will go extinct has become widespread enough to deserve its own acronym: Minimum Viable Population, or MVP (Harcourt 2006: 237). Reed and 
colleagues define an MVP as one that is large enough to ensure a 99 per cent probability of persistence for 40 generations. "In order to ensure long-term persistence of vertebrate populations," they claim, "sufficient habitat must be conserved to allow for approximately 7,000 breeding age adults" (Reed et al. 2003: 30). Other conservation biologists present other numbers, and yet others question the supposition that one can effectively use a single threshold of population size to indicate the level of threat for all nonhuman species, arguing instead for species-by-species thresholds (Harcourt 2006: 241; see also Pacheco 2004: 1178-79).

But while many conservation biologists agree that there is no magic number that represents a viable population size for all species, they also agree that for a population to be viable it must be "as numerate and as quantitative as possible" (Ballou, Gilpin, and Foose 1995: 277). Such an emphasis on numbers has become, for many scientists, the ultimate measure of viability. Calculations based on statistical norms to measure demography, threat level, and mortality are all tied to the governance of populations and, in this sense, are distinctly biopolitical. Foucault defines biopower as: "a power that exerts a positive influence on life, that endeavors to administer, optimize, and multiply it, subjecting it to precise controls and comprehensive regulations" (Foucault 1990: 137). At stake, then, "is the biological existence of the population" (ibid.). The management of animal populations for conservation is, indeed, "situated and exercised at the level of life, the species, the race, and the large-scale phenomena of populations" (ibid.).

\section{Algorithmic Life}

Algorithms are inert, meaningless machines until paired with databases upon which to function.

$$
\text { Gillespie (forthcoming) }
$$

The paradigm of viability-namely, the essence of population life, as distinct from the life of the individual-translates in conservation biology into a series of complex algorithms that quantify various characteristics of a population. This, with the goal of projecting the risk of extinction $(\mathrm{E}=1.0)$ within a particular timeframe. In the broadest sense, algorithms are "encoded procedures for transforming input data into a desired output, based on specified calculations" (Gillespie, forthcoming). Algorithms such as $((\mathrm{A}=1) * 5)+((\mathrm{A}>=2) * 95)$ for breeding analysis and $(0.2) * 1+(0.8) * 2$ for offspring distribution are the bread and butter of viability calculations in conservation biology (Sonoran Pronghorn PHVA 2013; see, e.g., Figure 5). Ballou explains the potential effects of such calculations:

If you're managing your health, and you do a viability analysis, and it says: "Hey, you've got a fifty per cent chance of dying in five years, and it's because you smoke too much"then you will go: "Ah, so my PVA - my evaluation of my health-tells me I can change my probability of extinction by stopping to smoke."

(Ballou, interview) 


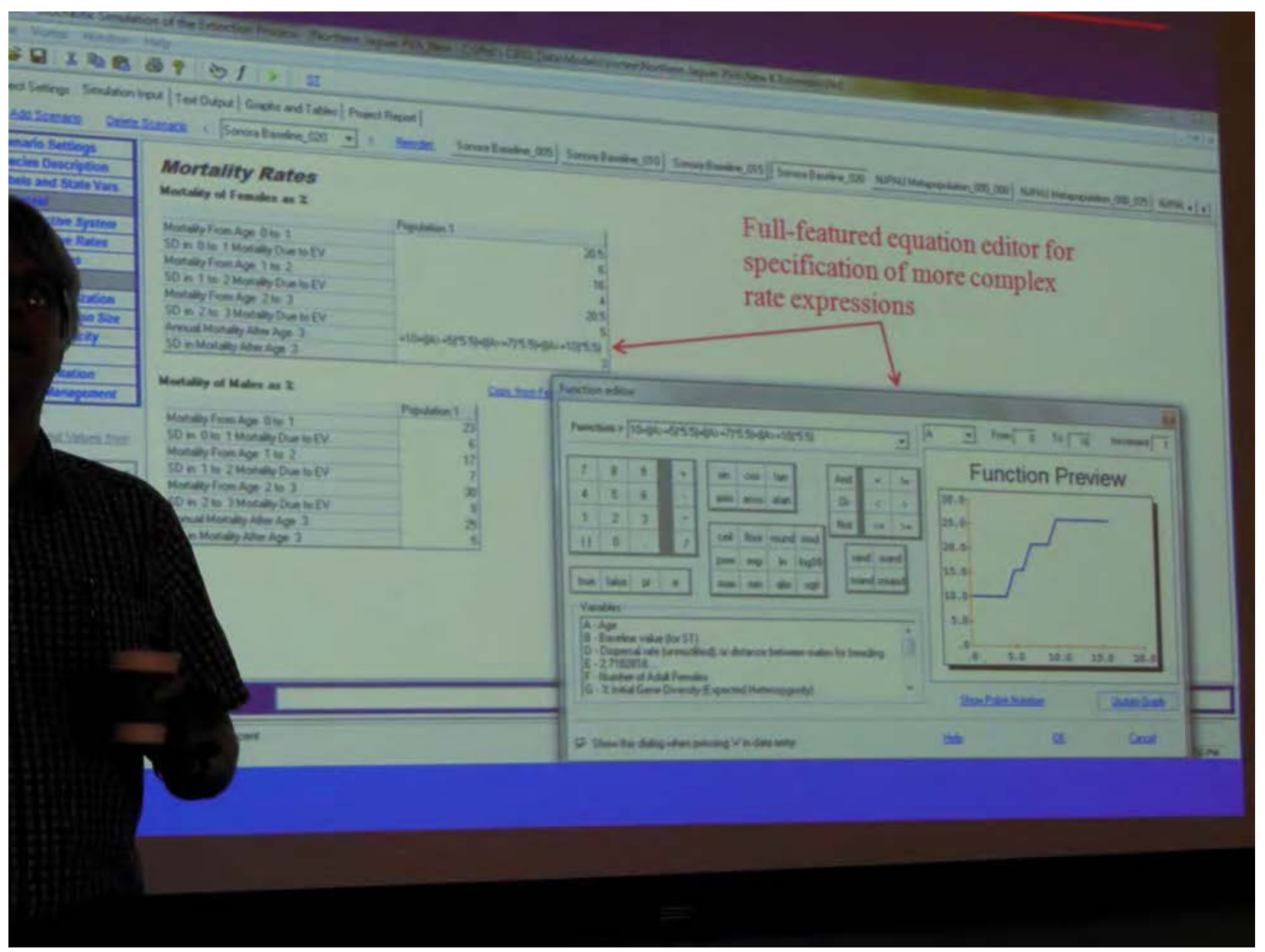

Figure 5: Phillip Miller of CBSG translating data into mortality rates, Sonoran pronghorn PHVA, April 22-24, 2013. Photo by author.

The life and death of populations have thus become the products of precise algorithmic calculations. Max Horkheimer and Theodor Adorno have suggested in a different context that:

When in mathematics the unknown becomes the unknown quantity in an equation, it is made into something long familiar before any value has been assigned. Nature, before and after quantum theory, is what can be registered mathematically; even what cannot be assimilated, the insoluble and irrational is fenced in by mathematical theorems. In the preemptive identification of the thoroughly mathematized world with truth, enlightenment believes itself safe from the return of the mythical.

(Horkheimer and Adorno 2002: 18)

While I agree with their general point about nature's translation into a series of mathematized registers, I take issue with Horkheimer's and Adorno's argument that the mathematization process is inevitably also one of demystification. My fieldwork has demonstrated, to the contrary, that the transformation of data into future predictions, when occurring through algorithmic configurations, is a highly specialized enterprise that is viewed by other experts with awe, as if it were alchemy. After three days of data gathering from twenty or so experts who work on the recovery of the Sonoran pronghorn antelope in Arizona and Mexico (see Figure 6, overleaf), Phillip Miller of the CBSG used algorithms to transform the data into a series of graphs and charts. The end result was presented by Miller to an entranced audience: although everyone knew the data as much, if not better, than Miller, no one could predict how thousands 
of simulations would affect the data. As it were, the result was quite clear: $E=1.0$ (see Figure 7, overleaf). This, it was clear to everyone in the room, meant that under the current management plan, the pronghorn will become extinct within a few decades. This result came as a slap in the face to many in the room who have dedicated their lives to saving the pronghorn, triggering a much-heated debate. Clearly, something significant had happened during the algorithmic process. But what was it?

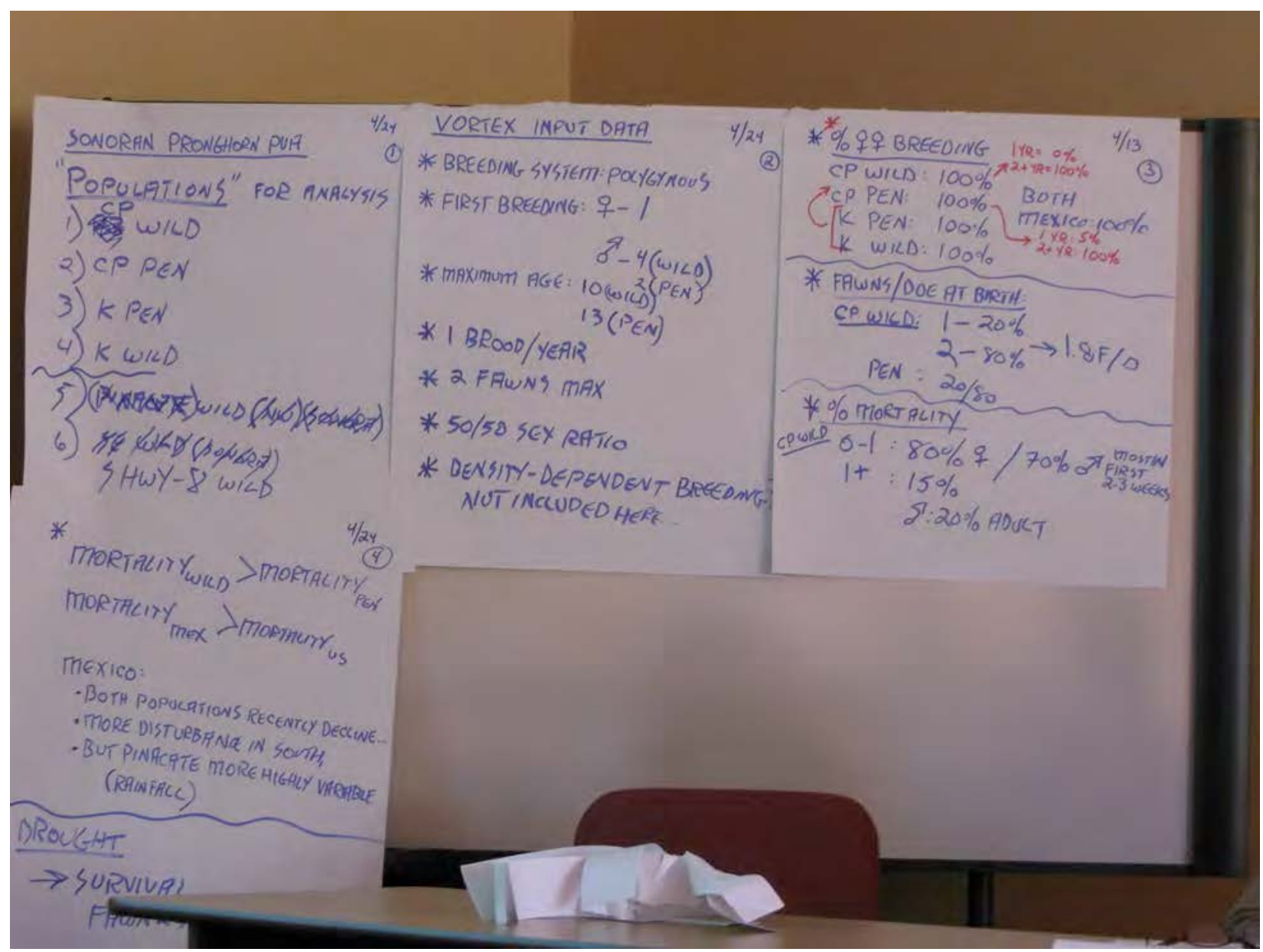

Figure 6: Collecting data, first day of Sonoran pronghorn recovery team meeting. Tucson, Arizona. Photo by author, April 22, 2013.

Although Gillespie's study of algorithms (Gillespie, forthcoming) draws on examples from mass communication technology, his work provides relevant insights for this project. Gillespie insists that algorithms operate upon data, and that they do so by selecting the most relevant data. The data is prepared for the algorithm, he argues, cleaned up so that the algorithms could then act upon it, seemingly automatically. Furthermore, "algorithms are also stabilizers of trust, practical and symbolic assurances that their evaluations are fair and accurate, free from subjectivity, error, or attempted influence" (ibid.). Indeed, the criteria and code of algorithms are generally obscured and their procedures hidden, although not equally so. There is something "impenetrable about algorithms. . . [T] hey are deliberately obfuscated, and they work with information on a scale that is hard to comprehend" (ibid.). In this sense, algorithms are black boxes for a new form of knowledge/power. During the Sonoran pronghorn workshop, it was only one population expert who knew how to work the algorithms. The other scientists and experts in the room did not question either the validity of the algorithmic process or the values embedded in them. Their only challenges were at the level of data accuracy and establishing what information had contributed most to the undesirable result. 


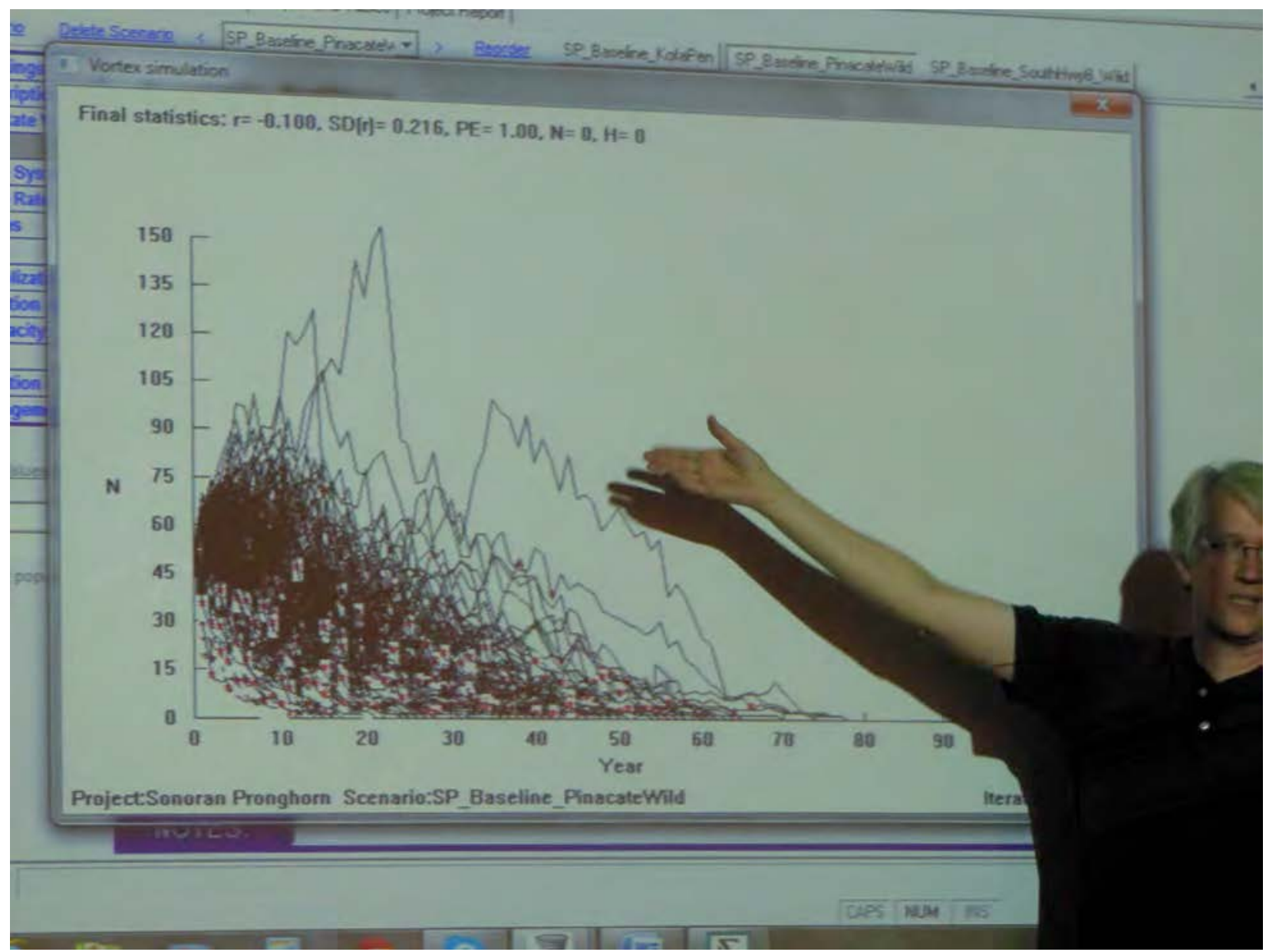

Figure 7: Phillip Miller of the CBSG presenting the results of the PHVA model at the Sonoran pronghorn recovery meeting in Tucson, Arizona. Photo by author, April 24, 2013.

\section{In Situ and Ex Situ Models: A Comparison}

The wild and captive computer models I have discussed share in common a concern with the management of small animal populations. Yet they are still different, as I will discuss here. Captive populations typically confront particular threats such as genetic inbreeding and drift, whereas small populations in the wild are more vulnerable to stochastic (random) and other events that are usually absent from controlled zoo settings. The goal of zoo animal programs is to identify and recommend reproductive choicesnamely, which pair of animals from which zoos should be mated to optimize gene diversity (Ballou, interview). Specifically, the major goal of captive management models has been to generate mating recommendations in terms of genetic sustainability through programs like ZooRisk, PM2000, and PMx. By contrast, in wild populations reproduction has traditionally not been controlled in the same fashion. As a result, the central focus of in situ population models has typically been demographic, relying upon the Vortex computer program.

Furthermore, while captive population managers calculate genetic diversity based on the assumption of 90 per cent for one hundred years, wildlife managers use PVAs to measure the viability of the population (Ballou). For the most part, genetic information has been unobtainable for wild populations and so their management has focused on calculating numbers and risk of extinction under PVAs. "Some of the PVA models don't even have a way to incorporate genetics," Ballou explains. "You could go back to a wildlife manager textbook in the 1980s and even the 1990s," he says, "and there would be no mention of genetic 
management at all." "But that's changed," he continues. "Now we know that wild populations can become small, can become inbred, can have problems - and there are plenty of examples of that." "Still," Ballou concludes, "there is a bit of an attitude in the wildlife community that genetics aren't as important in wild populations." "The majority of conservation biologists would certainly disagree with that," he finally states.

As stated, although it is central for both population models, the term viability takes on a very different meaning in each. Lees says: "There is no single definition of viability. You have to decide what you mean by it" (interview). With regard to wild population models, she adds, "we usually decide it in terms of an extinction probability over a defined period of time, because that's what we can measure" (Lees, interview). Still in the context of wild populations, Lees explains that:

Even though we know that species will all go extinct, eventually, what we're aiming for is perpetual life. What we are aiming for is immortality, really - or at least the trajectory of life that the species might have had if people had not stood in the way of that. . . In PVA models we look at how many times this population dies, or disappears below zero-or disappears below a number where we feel that it's going to start to lose its character as a species.

(Lees, interview, emphasis added)

On the other hand, for captive populations the calculations are typically carried out for shorter periods: "You look at what happened last year, and you decide what's going to happen next year . . And so you tend to look year by year," Lees explains. "Often," she continues,

the tools that we use for [captive management] don't have a stochastic element. They're a calculation rather than a simulation. The PVA was traditionally coined for a probabilistic analysis of extinction risk. With captive populations, we don't think of extinction risk because there are very few captive populations where there isn't also a wild population. So we don't tend to use the word "extinction," we just use words like "unsustainable."

(Lees, interview)

The in situ - ex situ divide in population management thus manifests in a parallel divide between demography and genetics, between viability and sustainability, and between simulation and calculation. Nonetheless, the current tendency in the science of small population management is toward integrating captive and wild systems by bringing them into one holistic management system. One example is IUCN's "One Plan" approach.

\section{The "One Plan" Approach}

Many conservation biologists believe that the idea of managing individual species in isolation is no longer realistic, in turn calling for a "metapopulation" management. The central idea behind this suggestion is that it is more likely for smaller and fragmented populations to be viable if they are managed interactively. "Any small, isolated population is at high risk of extinction. In a system of such small populations, the same factors of extinction still operate but not at the same time or in the same way. Hence, the overall system, the metapopulation, has a higher probability of survival" (Ballou, Gilpin, and Foose 1995: 280). To achieve viability goals, a metapopulation strategy recommends the number, size, and distribution of the subpopulations and the level of interchange among them (Ballou, Gilpin, and Foose 1995: 280).

The metapopulation concept, like many of the concepts discussed here, has also travelled between the in situ and ex situ worlds. Finnish ecologist Ilkka Hanski coined this term during his field observation of butterflies. Hanski realized that fragmented butterfly patches do not operate in isolation; instead, the 
corridors between these patches enable the creation of a metapopulation that ensures that if one patch goes extinct, the other patch would come up again quickly (Hanski 1998: 41; see also Hofer, interview). Heribert Hofer, director of the Leibniz Institute for Zoo and Wildlife Research, explains the novelty of the metapopulation discovery: "The naïve picture is that there is one population that is set up as a patch and another population in another patch and they're fragmented and they don't really talk to each other. This comes from the fairly static view of thinking of how speciation occurred in the first place" (Hofer, interview). However, "the fact is," he continues, "that populations actually talk to each other, that animals move between two corridors, and that there are quick extinction successions of local populations and a quick rebirth of the population." The understanding that seemingly disparate populations are in fact interconnected micro-species units in a larger macro-species system has recently been imported from field science into conservation biology. Now, human management purports to do for fragmented populations what populations have previously done for themselves: re-establish their interconnections.

In 1979, Ulysses Seal formed the Conservation Breeding Specialist Group (CBSG) to institutionalize the communication between in situ and ex situ conservation experts. The CBSG is one of 16 committees in the IUCN's Species Survival Commission, with over 350 members. It has been developing, testing, and applying an array of science-based tools and processes to assist species management. One of the tools employed by the CBSG is the use of neutral facilitators to moderate small working group sessions (PHVAs, not to be confused with PVAs). CBSG has assisted in the development of conservation plans involving over 240 species through more than 500 workshops held in 67 countries (CBSG 2013). CBSG scientists believe that "the success of the workshop is based on the cooperative process of dialogue, group meetings, and detailed modeling of alternative species and habitat management scenarios" (Orangutan PHVA Final Report 2004).

Onnie Byers, the current director of CBSG, coined the term "One Plan" to represent the approach that coordinates the disparate practices of in situ and ex situ conservation under one umbrella. Byers defines the One Plan as: "an integrated species conservation planning that considers all populations of the species, both inside and outside their natural range, under all conditions of management, involving all responsible parties, and engaging all available resources" (Byers, interview). "And that is just not what is currently happening," she tells me, "the only animals being planned for now are those in captivity" (Byers, interview). Christoph Schwitzer of the Bristol Zoo adds: "the One Plan approach essentially regard[s] the entire population of a species, no matter whether that is in the wild, in captivity, in a zoo, in a reserve, in a semi-reserve, or whatever." "Obviously," he continues, "the wild population (however you want to define wild) is the one that we want to ultimately conserve in that 'wild' state. But the lines become very blurry, and we want to acknowledge that: not just look at the wild population-whatever that is - but look at all the animals from a given species or a group of species in any population" (Schwitzer, interview, emphasis added). This statement reflects the erosion, in certain scientific quarters, of the wild/captive divide, in the interest of effective management for species and populations (Braverman 2014a, 2015).

The One Plan is not only about synchronizing animal populations through integrated metapopulation management; it is also about synchronizing the behavior of their respective human experts. Schwitzer explains: "It means bringing together all the experts that manage these populations." In addition to the people who work with these animals in the field, Schwitzer says, "we should also have people around the table who manage populations of that species in zoos as well as government officials." The purpose: "that all these people will be sitting at one table and can all bring their expertise together, rather than having these separate conservation planning initiatives" (Schwitzer, interview). The One Plan is thus effectively a one table approach (for potential problems with this approach see Braverman 2015).

As part of the movement toward integrative management, population biologists are currently tweaking computer models so that they may be applied across the captive/wild population divide. Specifically, population simulation models that were developed initially for in situ use - and Vortex in particular-are 
being modified to include modules for captive populations. "There's nothing to stop anybody from doing PVAs on captive populations," Ballou tells me. "In fact," he says, "the people at the Lincoln Park Zoo have a grant to pursue doing PVAs for all of the AZA sponsored populations" (interview). Lisa Faust of the Lincoln Park Zoo coordinates this pilot program, which applies the PVA model through ZooRisk to a select number of animal programs in zoos. She informed me in June 2013 that her team had already analyzed twenty zoo animal programs from the bear, parrot, and feline taxa (Faust, interview). Ballou explains that such viability models are "more comprehensive than just using the 90 per cent over a hundred years" model, which has been the central mode of calculation for zoo populations thus far (Ballou, interview).

Michael Hutchins, former director of animal programs for the AZA, mentions the black-footed ferret management as the best example for a successful integrative model:

At one time they were one of the most endangered mammals in the world: their population had gone down to just a handful of animals. But a captive breeding program was started soon after the wild population was discovered, and there are now animals going back and forth between the captive breeding program and different reintroduction sites. . . . So that's one of the closest examples of true metapopulation management, which involves a lot of discussion about genetics because you are trying to maintain the genetic variability of these populations by moving animals between them-and moving them between captivity and the wild and then back from the wild into captivity, too.

(Hutchins, interview)

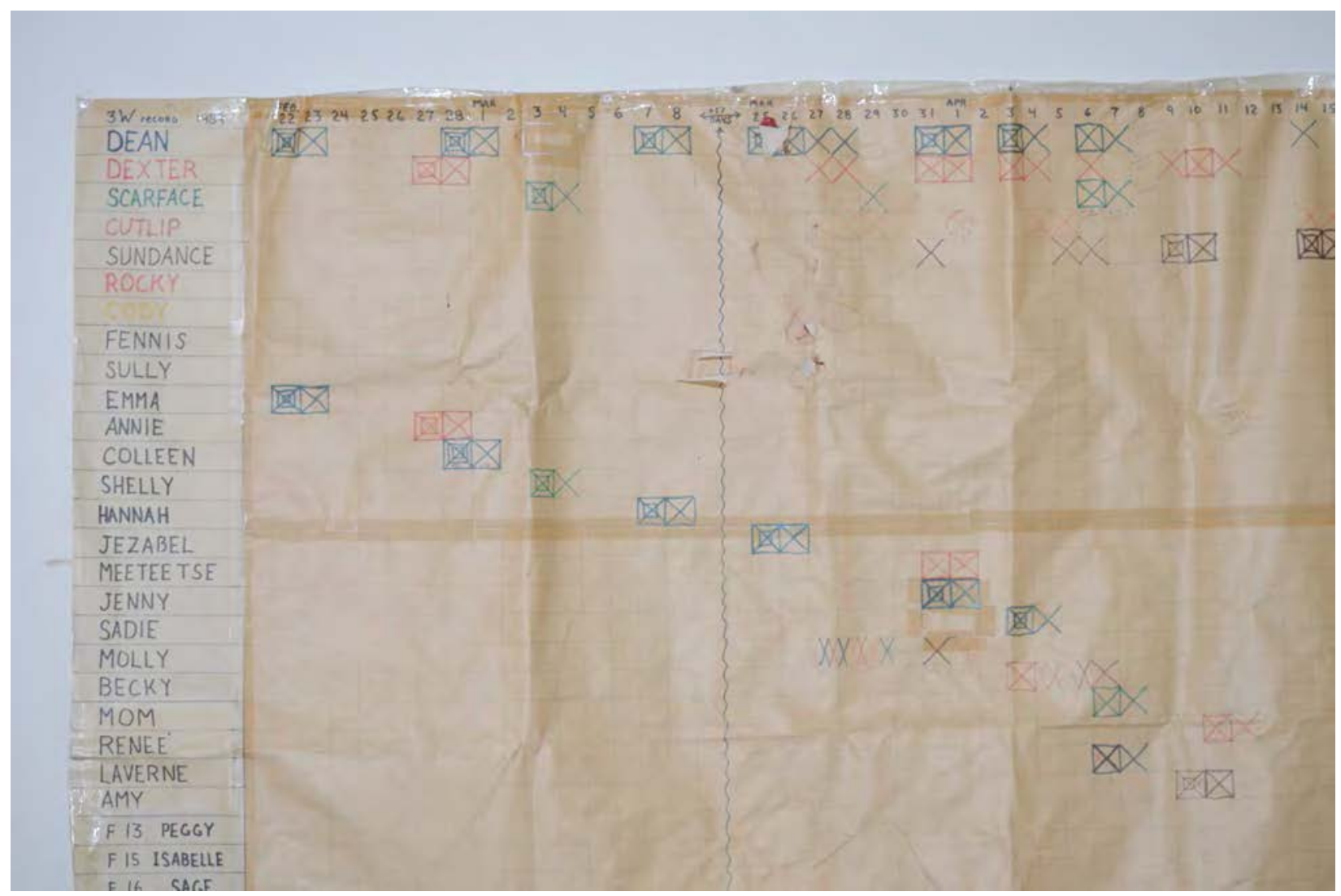

Figure 8: First genealogy chart created for the Black-footed ferret recovery program. Photo taken on July 31, 2012 by Ryan Moehring / USFWS 
Nonetheless, the metapopulation model has been quite controversial among scientists. "It comes back to the 'pure science' versus 'applied science' division," Lacy explains. "The Vortex modeling that I do is absolutely abhorred by some theoretical ecologists," he says, "who say it's getting down to the very specific and [that] it's not revealing the grand scheme of ecology." Rather than "Einstein-like grand equations that explain the universe," he argues, the kind of applied science promoted by the One Plan metapopulation approach "gets down to a whole lot of nitty-gritty natural history" (Lacy, interview).

\section{From Metapopulation to Meta-Models}

The metapopulation model enables a One Plan approach in contexts where there is more than one population of the same species or subspecies. Meta-modeling travels further down this managerial path. Phillip Miller explains the difference between metapopulation and meta-modeling: "A metapopulation model would use one single modeling tool, like Vortex, and would model within it multiple populations that interact demographically. A meta-model, on the other hand, links different models together" (Miller, interview). The individual, species-focused system of the single computer model thus serves as the foundation for the meta-model system.

The year 2013 saw the launching of the "MetaModel Manager" software program. This program is designed to link population demographics of multiple interacting species with other processes such as emerging diseases, habitat and climate change, landscape dynamics, and animal movement patterns. Lacy explains the significance of the meta-model approach in comparison to metapopulation systems of analysis:

The problem is that species do not exist in vacuums. The tools that were developed for doing very specific population modeling always made the implicit assumption that the rest of the world was static [and] that everything outside of the population can be treated as a constant. What we are moving toward is an approach of modeling where you cannot ignore the fact that what is outside the species is changing. So when you model a ferret you have to model a prairie dog and you have to model climate change-because the conservation problems arise from the interactions between those threats. So we are enacting an approach called "meta-model," where we are doing a fifty-year projection on the species model.

(Lacy, interview; see Figure 9)

To reiterate, meta-models now allow linkages between "small-scale, individual-based models (e.g., Vortex) and population-based models (e.g., RAMAS Metapop) in order to extend local analyses to the landscape scale or to understand interactions of species best modeled at the level of a single population with species best modeled at a broader, metapopulation scale" (Raboy and Lacy 2013: 13). From the program's manual: "The program runs iteratively and its results are generated in the form of means or probabilities, within the linked-program platforms. Analysis and interpretation of results can be used in the establishment of conservation goals and to direct or prioritize conservation action" (Raboy and Lacy 2013: 2). 


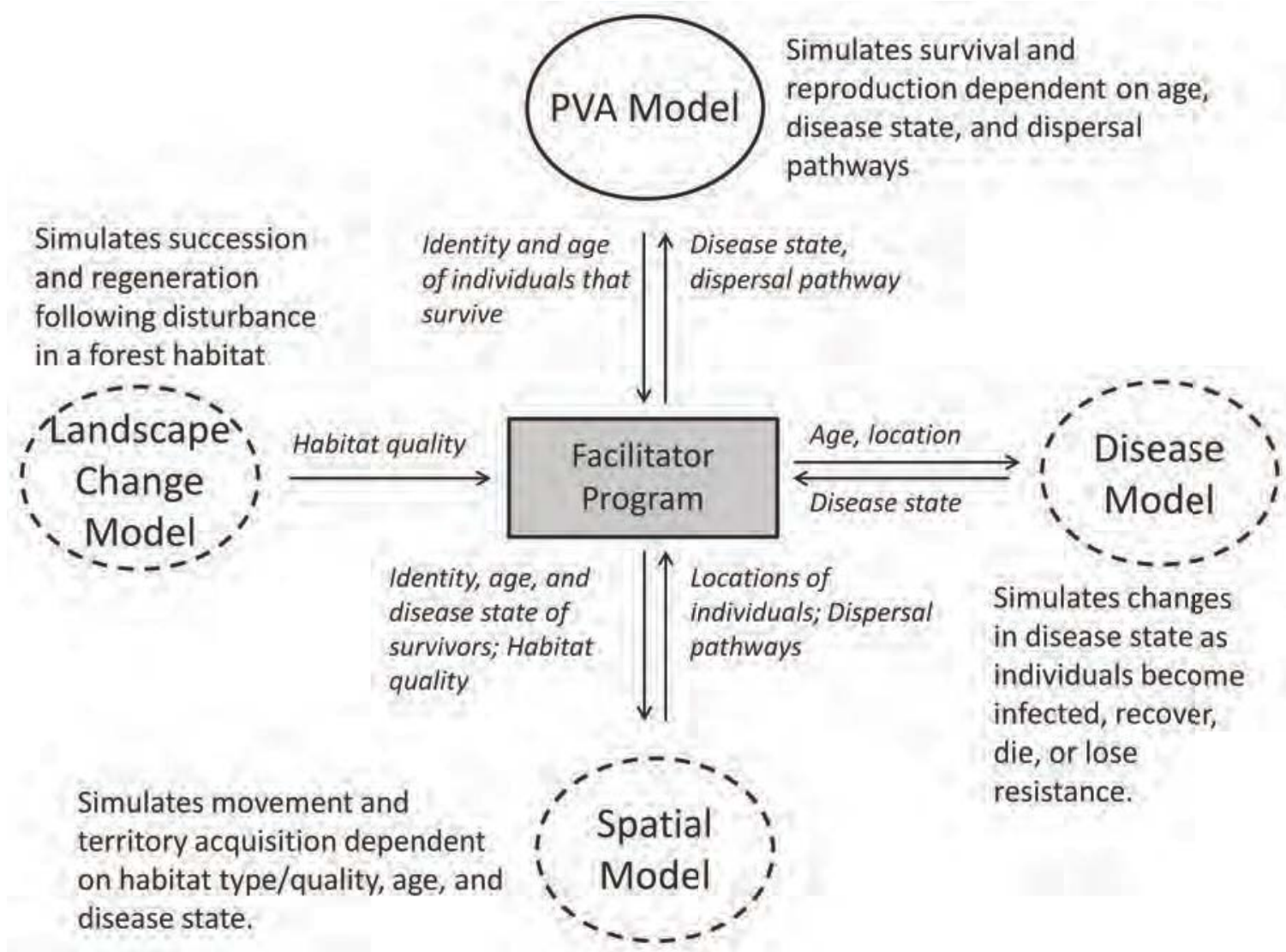

Figure 9: A graphic depiction of a meta-model that integrates demography, landscape change, dispersal, and disease status. "A PVA program acts as the system model to simulate individual survival and reproduction based on individual and population state variables (shown in italics) passed from other models. Modifier models (dashed outlines) simulate habitat dynamics, individual movements, and individual transitions in disease status. A central facilitator program passes state variables between the system and modifier models at appropriate time steps. The ultimate results are measures of population dynamics and extinction risk for a species impacted by habitat change and disease" (Lacy et al. 2013: 3). Used with permission.

I will draw on a hypothetical situation from an article by Lacy and colleagues (2013) to demonstrate the use of the meta-model system. In this hypothetical, an infectious disease is introduced to a metapopulation of a species consisting of ten subpopulations with a total population of $\mathrm{N}=250$. In the meta-model, population viability is predicted within the PVA software Vortex, while disease processes are simulated within the epidemiological model Outbreak, and these two model processes are linked via MetaModel Manager (Raboy and Lacy 2013: 13-14). Lacy and colleagues show how using the two models separately leads to contradictory results, whereas their integration takes into account the dual effects of synchronicity and disease. In their words:

Differences in the impacts of population connectivity on metapopulation dynamics in the absence [Figure 10, A] or presence [Figure 10, B] of disease illustrates the opposing forces acting on sub-divided populations: stochastic processes threaten small, isolated populations and are countered by dispersal while infectious disease transmission threatens interconnected populations and is blocked by isolation. Both processes impact many real populations, but the balance between these forces would be difficult to predict from the application of standard PVA methodology for population modeling, from standard 
epidemiological models of disease, or even from the examination of both models independently.

(Lacy et al. 2013: 7)
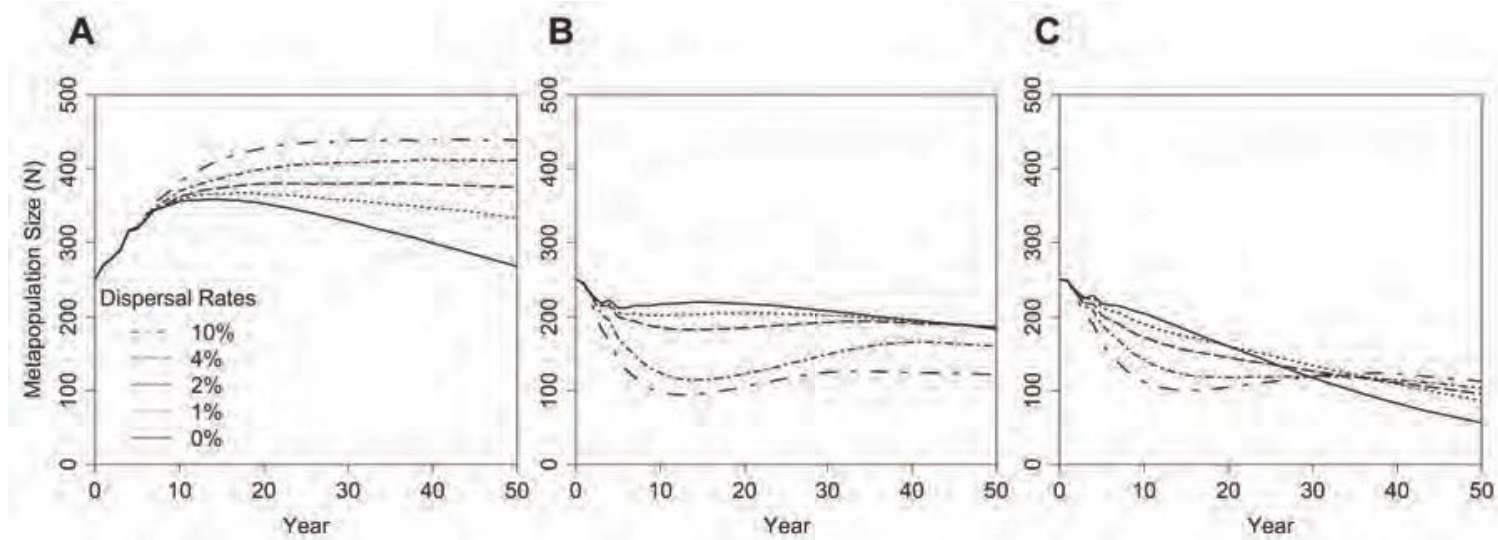

Figure 10: Whereas graphs $A$ and $B$ illustrate the limits of the metapopulation model, graph $C$ illustrates the benefits of the meta-model approach. From Lacy et al.: "Metapopulation size is projected by (A) a PVA model in Vortex assuming varied rates of dispersal, annual fluctuations in demographic rates, and inbreeding depression; (B) a metamodel that linked a PVA model in Vortex to an infectious disease model in Outbreak, assuming varied rates of dispersal, minimal annual fluctuations in demographic rates, and no inbreeding depression; and (C) a metamodel that linked a PVA in Vortex to an infectious disease model in Outbreak, assuming varied rates of dispersal, annual fluctuations in demographic rates, and inbreeding depression." (Lacy et al. 2013: 7). Used with permission.

And yet there is much that is still not accounted for in these models, Ballou tells me, sketching what he believes are the future steps for integrating in situ and ex situ management models. The next step, according to Ballou, is to figure out the relevance of metapopulation models for herd, flock, and group management. In his words,

We have really good tools to manage individuals, where we know their pedigrees. But we don't have good concepts or tools yet to manage groups. [For example,] if you have fifty fish tanks, and you have fifty fish in each tank, or you have flocks of birds, or you have herds of animals, where you don't necessarily know the individuals and you can't necessarily manage matings within the group. ... Those tools will then be very useful for managing wild populations, especially where you may have fragmented populations.

(Ballou, interview)

Ballou mentions the golden lion tamarins as an example of a fragmented population that would benefit from such an analysis. "There are as many as 17 different isolated populations," he says, arguing that "we're going to need to come up with a strategy for moving animals around so that small populations don't get too inbred, or die out." "That," he concludes, "is definitely going to be a place where the concepts for management are going to be very similar between wild and captive populations" (Ballou, interview). This, then, will enable a governance of populations that obviates the need to know individuals.

\section{Conclusion}

My essay has illuminated a few biopolitical characteristics of conservation biology. It has examined how changing conceptions of nature have justified an increasingly complex series of algorithmic calculations and predictions and, vice versa, how such calculations have led to different conceptions of nature and 
species conservation. The modelers of these new regimes are conservation biologists who are motivated by the aim of increasing viability and preventing extinction.

Specifically, the essay has traced the evolution of animal databases and population management models: from the separate management of species - in controlled ex situ settings through software programs such as ZooRisk and in in situ settings through Population Viability Analysis (PVA) models - to integrative metapopulation and meta-models that enable the management of small animal populations across disparate locations. I have focused especially on one integration model: the One Plan approach.

I have dedicated considerable attention to the growing importance of algorithms. I have argued, in particular, that the goal of conservation biologists when developing population models is to translate individual bodies and socio-material phenomena into condensed algorithmic relations that then enable calculated projections of risk. Mary Beth Mader writes: "it is the properties endogenous to mathematical objects - continuous quantity, the basic laws and operations of arithmetic - that create the alleged comparability and continuities of populations and other social phenomena" (Mader 2007: 20). When expressed as algorithms, means, and averages, material relations between animate bodies are objectified and abstracted, resulting in a power-knowledge nexus that is perceived to be insulated from the social world (Mader 2007: 21). Mader observes, along these lines, that this mathematical expression is "so ontologically alien to the social world that it fails to quality [sic] as a relation at all" (ibid.). I have demonstrated how algorithmic configurations also alienate a range of other conservation experts, who in turn become outsiders to this process, or mere suppliers of data. Drawing on the particular genealogy of the in situ - ex situ relationship in conservation biology, this essay has only just begun to unravel the algorithmic properties of biopower so as to "anti-program" them back into society (Latour 1999: 166), and to show how inescapably social and how highly immersed in particular epistemological practices they really are.

\section{Acknowledgments}

I would like to thank Phillip Miller, Robert Lacy, Jonathan Ballou, Onnie Byers, and William Waddell for their incredible patience and for their support of this project. My gratitude also extends to John Schlegel, Peter Steeves, Kevin Haggerty, and the anonymous reviewer of this journal, as well as to my colleagues at Cornell University's Society for the Humanities. Research for this project was funded by the American Council of Learned Societies' Charles A. Ryskamp Research Fellowship and by the Baldy Center for Law \& Social Policy.

\section{Interviews}

Stephen Amstrup (Director, Polar Bears International), interview by author, telephone, June 26, 2013.

Jonathan (Jon) Ballou (Research scientist, Smithsonian Institute), interview by author, telephone, June 6, 2013.

Lisa Faust (Vice president of conservation and science, Lincoln Park Zoo), interview by author, telephone, June 20, 2013.

Heribert Hofer (Director, Leibniz Institute for Zoo and Wildlife Research), in person, Disney, Florida, October 11, 2013.

Leslie Honey (Vice president of conservation services, NatureServe), interview by author, telephone, July 12, 2013.

Michael Hutchins (Former director, Animal Programs, American Association of Zoos and Aquariums; Director, Conservation and Science, Safari Professionals), interview by author, telephone, January 18, 2013, and in person, Washington DC, June $10,2013$.

Robert Lacy (Senior conservation scientist, Chicago Zoological Society/Brookfield Zoo), interview by author, telephone, July 31 , 2012, and in person, Disney, Florida, October 11, 2013.

Phillip Miller (Senior program officer, Conservation Breeding Specialist Group - CBSG), interview by author, in person, Tucson, AZ, April 22-24, 2013 and by Skype, May 6, 2013.

Karin Schwartz (Biological database manager, Chicago Zoological Society (Brookfield Zoo)), interview by author, telephone, April 1, 2013.

Christoph Schwitzer (Head of research, Bristol Zoo; Vice president of captive care and breeding, International Primatological Society; Adviser, Primate Specialist Group, IUCN), interview by author, Skype, May 5 \& 13, 2013.

William Waddell (Red wolf species coordinator, Red Wolf SSP, AZA; Point Defiance Zoo), interview by author, June 6, 2013.

\section{References}

Ballou, J.D., M. Gilpin and T.J. Foose. 1995. Population Management for Survival \& Recovery: Analytical Methods and Strategies in Small Population Conservation. New York: Columbia University Press. 
Bowker, G. 2000. Biodiversity Dataversity. Social Studies of Science 30 (5): 643-83.

Braun, B. 2006. Towards a New Earth and a New Humanity: Nature, Ontology, Politics. In: David Harvey: A Critical Reader, eds N. Castree and D. Gregory, 191-222. Oxford: Blackwell.

Braverman, I. Forthcoming, 2015. Wild Life: The Nature of In Situ and Ex Situ Conservation. Stanford: Stanford University Press.

2014a. Conservation without Nature: The Trouble with In Situ versus Ex Situ Conservation. Geoforum 51: 47-57.

2014b. Captive for Life: Conserving Extinct Species through Ex Situ Breeding. In: The Ethics of Captivity, ed. Lori Gruen. Oxford: Oxford University Press.

2012a. Zooland: The Institution of Captivity. Stanford: Stanford University Press.

2012b. Zooveillance: Foucault Goes to the Zoo. Surveillance \& Society 10 (2): 119-133.

Burney, D.A. and L. Pigott Burney. 2010. Inter situ conservation: Opening a 'third front' in the battle to save rare Hawaiian plants. BGjournal 6(1): 16.

CBSG 2013. CBSG History. Accessed March 9, 2014. http://www.cbsg.org/about-cbsg/history

Faust, L. and J.M. Earnhardt. 2008. Zoo Risk: A Risk Assessment Tool; Version 3.8, User's Manual. Chicago: Lincoln Park Zoo.

Fish and Wildlife Service. 2013. Red Wolf Recovery Program. Accessed March 6, 2014. http://www.fws.gov/redwolf

Foucault, M. 1990. The History of Sexuality: An Introduction, Vol. I. New York: Vintage.

Friese, C. 2013. Cloning Wildlife: Zoos, Captivity, and the Future of Endangered Animals. New York: NYU Press.

Gillespie, T. Forthcoming. The Relevance of Algorithms. In: Media Technologies, eds T. Gillespie, P. Boczkowski and K. Foot. Cambridge, MA: MIT Press.

Haggerty, K. and D. Trottier. 2013. Surveillance and/of Nature: Monitoring Beyond the Human. Society \& Animals (January 2013): 1-20. Accessed March 4, 2014. doi: 10.1163/15685306-12341304.

Hanski, I. 1998. Metapopulation dynamics. Nature 396 (6706): 41-49.

Haraway, D. 2008. When Species Meet. Minneapolis: University of Minnesota.

Harcourt, A.H. 2006. Empirical Estimates of Minimum Viable Population Size for Primates: Tens to Tens of Thousands? Animal Conservation 5 (3): 237-244.

Hinchliffe, S. and S. Whatmore. 2006. Living Cities: Toward a Politic of Conviviality. Science as Culture 15 (2): $123-138$.

Holloway, L. and C. Morris. 2012. Contesting genetic knowledge-practices in livestock breeding: biopower, biosocial collectivities and heterogeneous resistances. Environment and Planning D: Society and Space 30: 60-77.

Horkheimer, M. and T. Adorno. [1944] 2002. Dialectic of Enlightenment. Translated by Edmund Jephcott. Stanford: Stanford University Press.

Lacy, R., P. Miller, P. Nyhus, J.P. Pollak, B. Raboy and S. Zeigler. 2013. Metamodels for Transdisciplinary Analysis of Wildlife Population Dynamics. PLoS ONE 8(12): e84211. doi:10.1371/journal.pone.0084211

Latour, B. 1999. Pandora's Hope: Essays on the Reality of Science Studies. Cambridge, MA: Harvard University Press.

Lorimer, J. 2012. Multinatural geographies for the Anthropocene. Progress in Human Geography 36 (5): 593-612.

Mader, M.B. 2007. Foucault and Social Measure. Journal of French Philosophy 17 (1): 1-25.

NatureServe. 2011. National Species Dataset. Filed with author.

Orangutan PHVA Final Report. 2004. January 15-18. Accessed February 20, 2004. http://awsassets.panda.org/downloads/orangutanphva04_lowres.pdf, 12-14.

Pacheco, L.F. 2004. Large Estimates of Minimum Viable Population Sizes. Conservation Biology 18 (5): $1178-1179$.

Polar Bear Specialist Group. 2009. Mark-Recapture. Accessed March 20, 2014. http://pbsg.npolar.no/en/methods/markrecap.html.

Porter, T.M. 1996. Trust in Numbers: The Pursuit of Objectivity in Science and Public Life. NJ: Princeton University Press.

Raboy, B.E. and R.C. Lacy. 2013. Metamodel Manager, User's Manual. Brookfield, IL: Chicago Zoological Society.

Red Wolf Recovery Program. 2013. First Quarter Report, October-December 2013. Accessed March 6, 2014. http://www.fws.gov/redwolf/Images/20140128_RedWolf_QtrReport_FY14-01.pdf

Redford, K.H., D.B. Jensen and J.J. Breheny. 2013. The Long Overdue Death of the Ex Situ and In Situ Dichotomy in Species Conservation. WAZA Magazine 14: 19-22.

Reed, D. H, J.J. O'Grady, B.W. Brook, J.D. Ballou and R. Frankham. 2003. Estimates of Minimum Viable Population Sizes for Vertebrates and Factors Influencing those Estimates. Biological Conservation 113 (1): 23-34.

Shaffer, M. 1987. Minimum Viable Populations: Coping with Uncertainty. In: Viable Populations for Conservation, ed. Michael Soulé, 69-86. Cambridge: Cambridge University Press.

Shukin, N. 2009. Animal Capital: Rendering Life in Biopolitical Times. Minneapolis: University of Minnesota Press.

Smith, M. 2011. Against Ecological Sovereignty: Ethics, Biopolitics, and Saving the Natural World. Minneaopolis: University of Minnesota Press.

Smyth, B. and S. Nebel. 2013. Passive Integrated Transponder (PIT) Tags in the Study of Animal Movement. Nature Education Knowledge 4 (3): 3.

Sonoran Pronghorn PHVA. 2013. May 24-29, participatory observations by author.

Wolfe, C. 2013. Before the Law: Humans and Other Animals in a Biopolitical Frame. Chicago: University of Chicago Press.

Youatt, R. 2008. Counting Species: Biopower and the Global Biodiversity Census. Environmental Values 17 (3): $393-417$. 